\title{
Reduced Forebrain Serotonin Transmission is Causally Involved in the Development of Compulsive Cocaine Seeking in Rats
}

\author{
Yann Pelloux*,',2, Ruth Dilleen', Daina Economidou', David Theobald' and Barry J Everitt' \\ 'Behavioural and Clinical Neuroscience Institute and Department of Experimental Psychology, University of Cambridge, Cambridge, UK; ${ }^{2}$ Institut \\ dela Timone, CMRS UMR 7289, Marseille, France
}

\begin{abstract}
Whereas the majority of cocaine users quit as they experience the negative consequences of drug use, some lose control over their drug taking and compulsively seek drugs. We report that $20 \%$ of rats compulsively seek cocaine despite intermittent negative outcomes after escalating their cocaine self-administration. This compulsive subgroup showed marked reductions in forebrain serotonin utilization; increasing serotonin transmission reduced their compulsive cocaine seeking. Depleting forebrain serotonin induced compulsive cocaine seeking in rats with a limited cocaine taking history; this was reversed by systemic treatment with a 5-hydroxytryptamine (5-HT2C) receptor agonist and mimicked by systemic treatment with a $5-\mathrm{HT} 2 \mathrm{C}$ receptor antagonist in intact animals. These results indicate the causal involvement of reduced serotoninergic transmission in the emergence of compulsive drug seeking after a long cocaine-taking history.

Neuropsychopharmacology (2012) 37, 2505-25I4; doi:I0.1038/npp.20I2.III; published online 4 July 2012
\end{abstract}

Keywords: addiction; cocaine; compulsivity; punishment; serotonin; 5-HT2C receptors

\section{INTRODUCTION}

The majority of individuals who begin taking addictive drugs are eventually able to reduce their intake and become abstinent; a major reason for doing so is to avoid the negative consequences of chronic drug use (Waldorf et al, 1991). In contrast, a smaller proportion of individuals who initially use drugs, generally estimated to be about $20 \%$ (Anthony et al, 1994), ultimately lose control over their drug taking and compulsively seek drugs. This compulsive drug use is defined as the maladaptive propensity to repeat, or perseverate, in drug seeking or consumption even in the face of significant aversive or disadvantageous consequences (Waldorf et al, 1991; Ersche et al, 2011; American Psychiatric Association, 2000).

In order to investigate the mechanisms involved in the emergence of compulsive drug seeking, novel animal models have been developed that are based upon the persistence of drug seeking or taking despite negative, or aversive, outcomes (Heyne and Wolffgramm, 1998; Spanagel and Holter, 1999; Panlilio et al, 2003; Deroche-Gamonet et al, 2004; Vanderschuren and Everitt, 2004; Cooper et al,

\footnotetext{
* Correspondence: Yann Pelloux, Institut des Neurosciences de la Timone, CNRS UMR 7289, 27 Blvd Jean Moulin, I 3385 Marseille cedex 05, France, Tel: 003349|324010,E-mail: yann.pelloux@univ-amu.fr Received I March 2012; revised 17 May 2012; accepted 27 May 2012
}

2007; Pelloux et al, 2007). These models have good face and ecological validity as they provide the opportunity to study both the compulsive aspect of addictive behavior as well as 'abstinence' and relapse in a way that does not require physical removal of the animal from the drug-taking environment for varying periods of time, or the extinction of instrumental drug-taking responses (Shaham et al, 2003). Previous studies on the punishment of cocaine seeking or taking have identified a subpopulation of about $20 \%$ of rats that becomes resistant to negative outcomes and therefore compulsively seek cocaine, but only after an extended, not a brief, cocaine-taking history (Deroche-Gamonet et al, 2004; Pelloux et al, 2007).

Studies of drug addicted humans and animals in experimental models of addiction have demonstrated neural adaptations that are correlated with, or caused by, chronic stimulant self-administration (SA). Although longterm changes in dopaminergic systems (Wilson et al, 1996; Porrino et al, 2007), especially a reduction in striatal D2 dopamine receptor binding, which is itself correlated with reduced basal metabolic activity in the orbital prefrontal cortex (Volkow et al, 2001), are well known, altered indices of serotonin (5-hydroxytryptamine (5-HT)) transmission have also been reported in addicted individuals. Cocaine and alcohol addicts have increased brain 5-HT transporter availability (Little et al, 1998; Jacobsen et al, 2000), while 5-HT levels in prefrontal 
cortical areas have been shown to be reduced in cocaine addicts postmortem (Wilson et al, 1996). These have been linked with deficits in behavioral inhibition that might underlie compulsive drug taking. Thus, chronic cocaine use in humans is associated with perseveration, a form of compulsive behavior, in a reversal learning task (Ersche et al, 2008), as well as impaired decision-making in a gambling task (Rogers et al, 1999a). Both deficits can be induced by depletion of brain 5-HT in healthy human volunteers (Rogers et al, 1999a; Rogers et al, 1999b). These data suggest a causal relationship between 5-HT transmission and the compulsive behavior seen in cocaine addiction (Ersche et al, 2008).

In the present study, we have investigated the serotoninergic correlates of compulsive cocaine seeking in rats using a procedure in which cocaine seeking results intermittently and unpredictably in mild punishment as well as the opportunity to take cocaine (Pelloux et al, 2007), thereby capturing the conflict between opponent motivational states that has been suggested to characterize addictive behavior (Waldorf et al, 1991; Ersche et al, 2011 and in DSMIV). We then tested the hypothesis that reductions in forebrain 5-HT are causally related to the development of compulsive drug seeking.

\section{MATERIALS AND METHODS}

\section{Subjects and Surgery}

The experimental procedures were conducted in accordance with the United Kingdom, 1986 Animals (scientific procedures) Act (project licence PPL 80/1767). Male Lister Hooded rats (180-200 $g$ at arrival; Charles River, Kent, UK) were housed in pairs, and food intake was maintained at $20 \mathrm{~g} / \mathrm{d}$. Two weeks after their arrival, rats were anesthetized with ketamine hydrochloride $(100 \mathrm{mg} / \mathrm{kg}$, i.p.; Ketaset) and xylazine ( $9 \mathrm{mg} / \mathrm{kg}$, i.p.; Rompun) implanted with a catheter (CamCaths, Cambridge, UK) and left to recover for one week, single-housed.

\section{Procedure}

Experiment 1. Rats were trained to acquire the 2-h seekingtaking (ST) heterogeneous chained schedule (random interval (RI) 120-s, FR 1) for cocaine reinforcement $(0.25 \mathrm{mg} /$ infusion at the rate of $0.1 \mathrm{ml} / 5 \mathrm{~s})$ with $10 \mathrm{~min} \mathrm{TO}$ between two ST cycles, as described previously (Pelloux et al, 2007). Briefly, after acquisition of the taking response for 5-7 days, animals were trained on the ST chain. Each cycle started with the insertion of the drug-seeking lever with the taking lever retracted, and the first press on the seeking lever initiated a RI schedule, which was progressively increased over 5 days $(2,15,30,60$, and $120 \mathrm{~s})$. The first lever press after the RI had elapsed resulted in the retraction of the seeking lever and insertion of the drugtaking lever. One press on the taking lever was followed by the drug infusion accompanied by the same stimulus events as during the training of the taking response. Each cocaine infusion was followed by a TO period, which was progressively increased across 4 sessions (20, 120, 240, 600 s). Thereafter, the seeking lever was reinserted to start the next cycle of the schedule.
Animals then underwent 12 days of $1 \mathrm{~h}$, for the short access (ShA) group, or $6 \mathrm{~h}$ (with a cutoff of 150 cocaine infusions), for the long access ( $\operatorname{LgA}$ ) groups, daily cocaine SA sessions with only the taking lever present (FR1, $\mathrm{TO}=20 \mathrm{~s}$ ) (see Ahmed and Koob, 1998). To investigate whether the motivation for cocaine was affected by cocaine history, we measured taking responses under a progressive ratio schedule and seeking responses in a probe test in which only the seeking lever was present, as described previously (see Pelloux et al, 2007). The assessment of performance in each of these tests was restricted to a single session to minimize any disruption of performance on the ST chain. During the progressive ratio test, the seeking lever was withdrawn and the ratio requirement of taking responses was increased after each reinforcer according to the following progression: $1,3,6,9,12,17,24,32,42,56,73$, $95,124,161,208,268,346,445,573$, and 737 . The value of the last ratio completed was taken as the break point (Hodos, 1961). The session ended after $4 \mathrm{~h}$ or after $40 \mathrm{~min}$ had elapsed after the previous reinforcer delivery. After the progressive ratio session, baseline performance on the ST chain was reestablished for two to three sessions before motivation and reinforcement were assessed using the seeking rather than the taking response. During this test session, the ST chain was suspended and responses on the seeking lever alone was measured (no cocaine was delivered). Completion of the seeking link led to a direct transition to the TO period, without the taking link, after which the seeking chain restarted (see Pelloux et al, 2007).

Both groups received three further sessions on the ST schedule during which rats were also trained to nose poke into the magazine for $0.04 \mathrm{ml}$ of a $20 \% \mathrm{w} / \mathrm{v}$ sucrose solution, which was delivered under a VI schedule, the parameter of which was progressively increased ( 2 and $15 \mathrm{~s}$ ) to $60 \mathrm{~s}$ on the third day, which was taken as the first day of baseline. This allowed for the assessment of any general suppression of appetitive behavior by, and thus the specificity of the effects of the introduction of, punishment of cocaine seeking. Three further sessions of training under the ST chain with concurrent sucrose reinforcement established a baseline against which were assessed the effects of punishment as described (Pelloux et al, 2007). During each punishment session, half of the cycles contained no punishment and were identical to those in baseline training. In the remaining cycles, the seeking response was ultimately punished, ie, the first response that met the RI requirement in the seeking link delivered a 0.5 -s foot shock and led to a direct transition to the TO period without the taking link. The reinforced and punished cycles were presented randomly within each session. Punishment sessions occurred daily for 8 consecutive days.

Each group of rats was the pool of two cohorts. After the 8 days of intermittent punishment the first cohort was subjected to a fear-conditioning procedure and assessed for the brain monoamine content whereas the effect of systemic administration of citalopram and atomoxetine on responding under intermittent punishment was evaluated in the second cohort (see Figure 1a).

Conditioned fear: Two minutes after introduction into the conditioning chamber, a clicker was presented for $1 \mathrm{~min}$, which ended with the administration of a single foot 

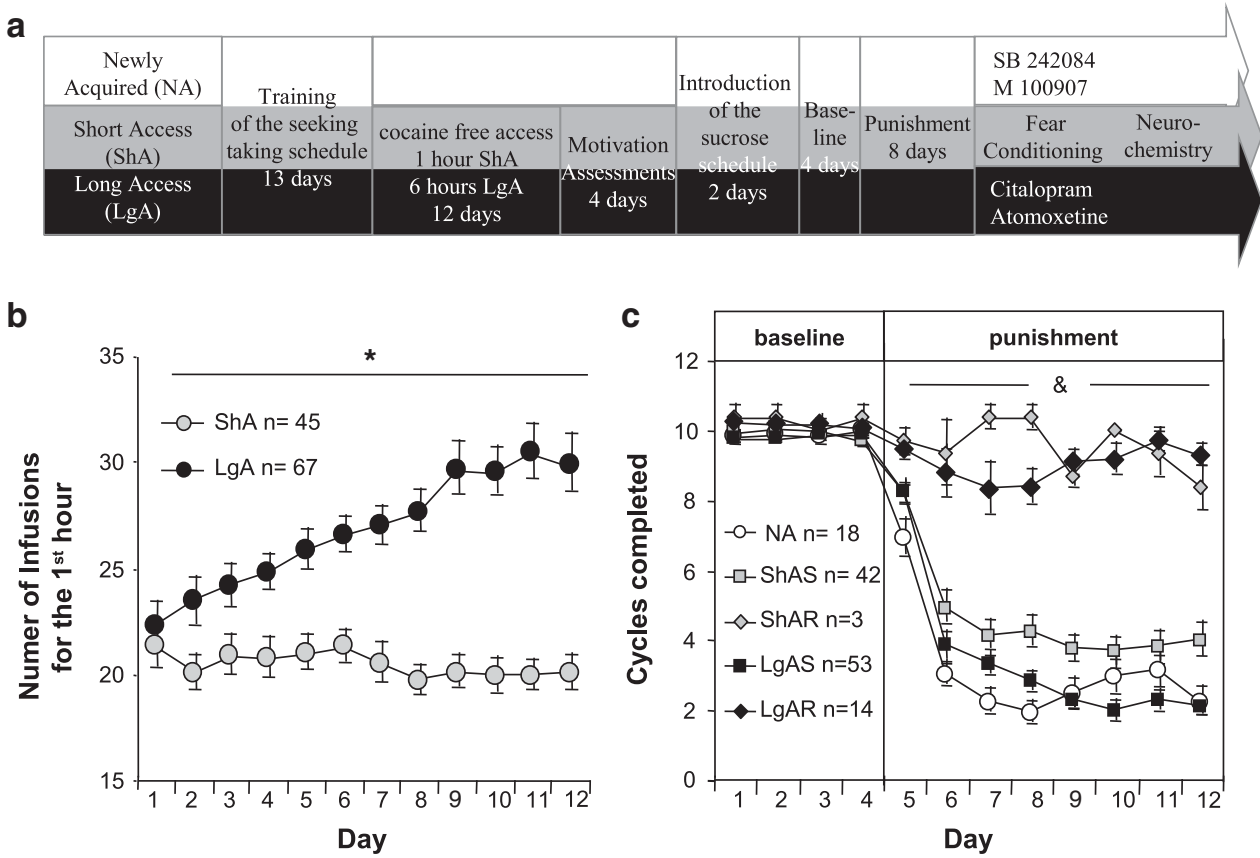

Figure I Emergence of a compulsive cocaine-seeking subpopulation of rats after an extended cocaine-taking history (a) Timeline of the experiment. (b) Early-loading phase during free access sessions, in animals having had 12 days of I h (ShA: gray dots) or $6 \mathrm{~h}$ (LgA: black dots) daily access to cocaine. Mean \pm SEM of 45 and 67 animals per group. Significant difference between ShA and LgA: *Fischer's LSD P<0.05. (c) Number of seeking cycles completed before (baseline) and during punishment of NA (white squares), ShA, and LgA sensitive (gray and black squares, respectively) or resistant (gray and black triangles). Mean \pm SEM of 3-53 animals per group. Significant differences between sensitive and resistant. "Fischer's LSD $P<0.05$.

shock $(2 \mathrm{~s}, 0.5 \mathrm{~mA})$. One minute later, the animal was returned to its home cage. Twenty-four hours later, the animal was returned to the conditioning chambers for a 20-min test session during which the clicker was alternatively switched on $(1 \mathrm{~min})$ and off $(1 \mathrm{~min})$. Freezing was scored every $5 \mathrm{~s}$ during the test session. For locomotor activity measurements of responsivity to foot shock, the cage was virtually divided into four equal sectors, and the number of sectors crossed (defined by the position of the base of the tail) was measured during the 2-s foot shock administration.

Measurement of monoamines and their metabolites in cortical and striatal structures: Approximately 1 week after the end of behavioral testing, animals were killed by $\mathrm{CO}_{2}$ induced asphyxiation and their brains removed to be sectioned $(150 \mu \mathrm{m})$ and punches of tissue $(0.75 \mathrm{~mm}$ diameter) removed from prefrontal and striatal regions as well as from the amygdala (Supplementary Materials Figure 1). Dopamine (DA), dihydroxypheylacetic acid (DOPAC), 5-hydroxytryptamine, 5-hydroxyindoleacetic acid (5HIAA), norepinephrine and methoxyhydroxyphenylglycol (MHPG) content were determined by reversed-phase high-performance liquid chromatography with electrochemical detection as described (Dalley et al, 2002)

After the 8 days of punishment, the most resistant animals $(n=5)$ that emerged after repeated cocaine exposure in the second cohort were treated with Citalopram (1, 3, and $10 \mathrm{mg} / \mathrm{kg}$; IP $1 \mathrm{ml} / \mathrm{kg}$, Tocris, UK), or with the selective norepinephrine reuptake inhibitor
(NARI), atomoxetine ( 1 and $3 \mathrm{mg} / \mathrm{kg}$; i.p. $1 \mathrm{ml} / \mathrm{kg}$, Lilly, UK) $20 \mathrm{~min}$ before testing according to a Latin-square crossover design.

Experiment 2. Following the analysis of experiment 1, we investigated the effect of posttraining forebrain 5-HT depletion on cocaine-seeking performance under punishment after a short history of cocaine. Animals were trained under the ST task, matched for baseline performance and divided into two groups. All rats were pretreated $30 \mathrm{~min}$ before with desipramine $\mathrm{HCl} \quad(15 \mathrm{mg} / \mathrm{kg}$, i.p.; Sigma Chemical, UK) to prevent reuptake of 5,7-dihydroxytryptamine (5,7-DHT) into, and thereby protecting, noradrenergic neurons from neurotoxic damage (Björklund et al, 1975). They were then anesthetized with volatile isoflurane (flow rate: $4 \%$ isoflurane in $0.2 \mathrm{l} / \mathrm{min} \mathrm{O}_{2}$; Novartis Animal Health UK, Herts, UK) and received stereotaxically bilateral injections of either 5,7-DHT creatinine sulfate $(80 \mu \mathrm{g}$ in $10 \mu \mathrm{l}$ per site, Sigma Chemical) or the vehicle $(10 \mu \mathrm{l}$ per site of $0.1 \%$ ascorbic acid in saline) aimed at the lateral ventricle (anteroposterior $-0.9 \mathrm{~mm}$ and mediolateral $\pm 1.5 \mathrm{~mm}$ from bregma, dorsoventral -3.5 from dura with the incisor bar set at $3.3 \mathrm{~mm}$ below the interaural line (Paxinos and Watson, 1996)).

After 10 days of recovery (Björklund et al, 1975), animals were re-baselined, assessed for their motivation for cocaine (for the first cohort), trained with concomitant nose-poke responding for sucrose under the cocaine ST schedule and maintained for 4 days under baseline against which the effects of punishment were measured. 
After 8 days of punishment, lesioned and control rats were treated with the 5-HT2C receptor agonist, $m$ chlorophenylpiperazine $(0.1,0.3$ and $1 \mathrm{mg} / \mathrm{kg}$ of $\mathrm{mCPP}$; i.p. $1 \mathrm{ml} / \mathrm{kg}$, Tocris, UK), $20 \mathrm{~min}$ before testing, across days according to a Latin-square crossover design, with 2 days of re-baseline between doses.

One week after the last day of punishment, animals underwent fear conditioning and 1 week later, animals were killed for the measurement of monoamines in the cortex, striatum, and amygdala.

Experiment 3. Based on the effect of mCPP to reverse 5-HT lesion-induced increases in cocaine seeking under punishment, we investigated further the effects of manipulating 5-HT transmission. As mCPP has affinity for the 5-HT2C and 5-HT2A receptor (Porter et al, 1999), we investigated the effects of systemic administration of the 5-HT2C antagonist SB 242084 (Solvay, Weesp, The Netherlands) and 5-HT2A antagonist M100907 (Solvay) or the vehicles (citric acid $25 \mathrm{mM}$, cyclodextrine $8 \%$ in $0.9 \%$ saline or saline, respectively) on performance under punishment in rats having recently acquired the cocaine ST task (NA). Eighteen rats were trained on the ST task with concomitant nose-poke responding for sucrose and maintained for 4 days on baseline responding against which the effects of punishment were assessed. After 8 days of punishment, performance was evaluated after injections of SB 242084 $(0,1,3,10 \mathrm{mg} / \mathrm{kg}$, i.p., $1 \mathrm{ml} / \mathrm{kg})$ or M100907 (0, 0.1, 0.1, $1 \mathrm{mg} / \mathrm{kg}$ i.p., $1 \mathrm{ml} / \mathrm{kg}), 20 \mathrm{~min}$ before testing across days according to a Latin-square crossover design.

\section{Statistical Analysis}

Data were analyzed using mixed analysis of variance with factors Group (Short Access (ShA) vs Long Access (LgA) vs Newly Acquired (NA) or Sham vs 5,7-DHT) and, where appropriate, with additional factors Resistance to Punishment (Sensitive vs Resistant) and Day, Phase (baseline vs punishment), Dose or Drug as repeated measures. Further analysis used Fischer's LSD post hoc tests when significant main effects or interactions warranted. Statistical dependence between variables was evaluated using Pearson's coefficient product $(\mathrm{r})$ or Spearman's rank correlation $(\rho)$ depending upon normality of population data.

\section{RESULTS}

During the sessions of free access to cocaine taking, rats with only $1 \mathrm{~h}$ daily access (ShA) had a stable and controlled pattern of cocaine intake over days (effect of day: $\mathrm{F}(11,484)=0.8)$, whereas daily 6 -h access (LgA) resulted in a progressive and significant escalation of cocaine intake (effect of day: $\mathrm{F}(11,726)=13 ; P<0.001$ ) (Figure $1 \mathrm{~b}$ ).

During intermittent punishment, rats having newly acquired the cocaine ST task (NA) showed a greater decrease in seeking responses than ShA and LgA groups (effect of punishment: $(\mathrm{F}(11,1397)=285 ; \quad P<0.001)$ Fischer's LSD post hoc ps <0.04) (Figure 1c). In addition, there was major variation in the distribution of individuals within the ShA and LgA groups in terms of the number of seeking cycles they completed during the last 4 days of punishment (Kolmogoroff Smirnoff $\mathrm{Z}=1.37, \quad P=0.047$; Supplementary Materials Figure 2). Indeed, rats in the LgA group fell into two sub-groups, one completing an average of $<$ six-seeking cycles (long-access punishment sensitive, LgAS) and one of just over $20 \%$ of rats (longaccess punishment resistant, LgA) completing on average $>8$, which corresponds to the lowest number of seeking cycles completed during baseline testing. Only 6\% of rats in the ShA group (short-access punishment resistant, ShAR, vs short-access punishment sensitive, ShAS) and none in the NA group did so (Figure 1c).

Animals resistant to seeking punishment in both the ShA and LgA groups had higher break points under a progressive ratio schedule of cocaine reinforcement (effect of resistance: $\mathrm{F}(1,108)=4.3 ; P=0.04$ ) and made more seeking responses in the probe test (effect of resistance: $\mathrm{F}(1,108)=4.7 ; \quad P=0.03)$ as well as under baseline conditions (effect of resistance: $\mathrm{F}(1,108)=5.2, P=0.02$ ) (Supplementary Materials Figure 3) and there were significant, correlations between resistance to punishment and these motivational measures (Spearman's $\rho$ 's $>0.326$ $P<0.001)$.

Nose-poke responding for sucrose during punishment did not decrease and even slightly increased (effect of punishment: $\mathrm{F}(1,125)=31.1 ; P<0.001)$, indicating that appetitive behavior was not generally suppressed by fear in the NA, ShA, and $\operatorname{LgA}$ punishment-sensitive rats (Supplementary Materials Figure 4).

Animals that varied in their resistance to punishment of cocaine-seeking responses showed no differences in their locomotor reactivity to the mild foot shock (effect of resistance: $F(1,42)=2.2$ ) or in their fear conditioning (effect of resistance: $\mathrm{F}(1,42)=0$ ) (Supplementary Materials Figure 5).

These punishment-resistant (compulsive) and punishment-sensitive animals formed the basis of further neurochemical investigation and analysis.

Serotonin turnover (5-HT/5-HIAA ratio) was significantly lower across prefrontal cortical and striatal areas as well as in the amygdala in both $\operatorname{ShA}$ and $\operatorname{LgA}$ rats that were resistant to punishment compared to those that were sensitive (effect of resistance: $\mathrm{F}(1,42)=6.7, P=0.01$ ); Figure 2a). In contrast, a reduction in DA turnover in the punishment resistant compared to sensitive rats was observed only in the dorsal striatum (effect of resistance: $\mathrm{F}(1,42)=3.9 ; \quad P=0.05$ ) (Figure 2b). There were no significant changes in NE turnover in any of the forebrain regions (Figure $2 \mathrm{c}$ ). There was no significant relationship between 5 -HT, DA or NE turnover and the motivational measures of break points under a progressive ratio schedule of reinforcement, responding during the seeking probe test or baseline seeking performance $(\rho \mathrm{s}(44)<0.21)$.

In the Resistant animals that emerged after extended cocaine taking from the second cohort, treatment with citalopram dose-dependently decreased the number of seeking cycles completed under punishment (effect of dose: $\mathrm{F}(3,12)=3.8, P=0.04$ ) (Figure $3 \mathrm{a}$ ) that was significant at the $10 \mathrm{mg} / \mathrm{kg}$ dose (Fischer's LSD $P=0.012)$ ). In contrast, atomoxetine was without effect on their responding under punishment (Figure 3b). Neither drug had any effect on concomitant responding for sucrose. 
a
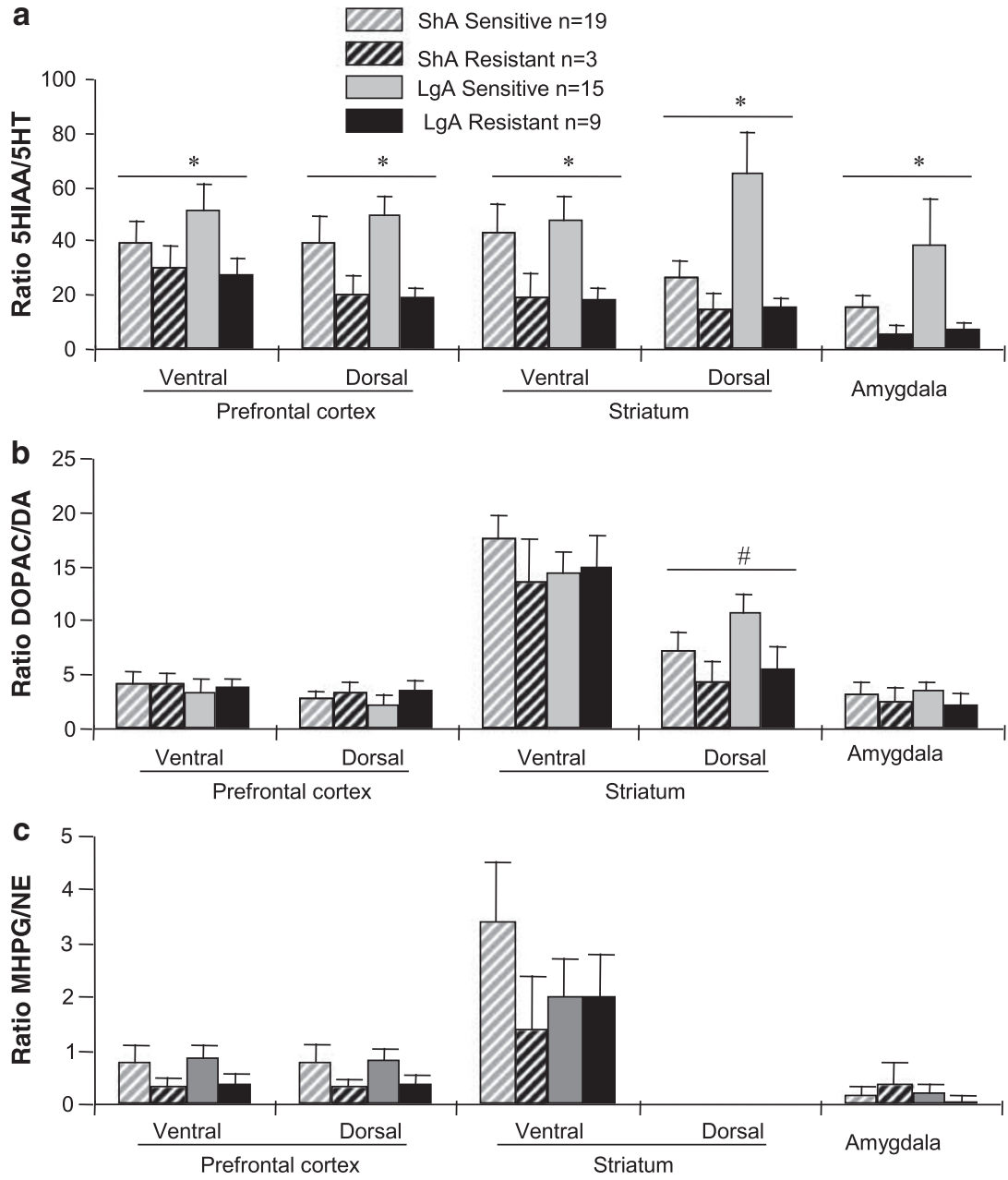

Figure 2 Neurochemical correlates of compulsive cocaine seeking. Utilization of 5-HT (panel a), DA (b) and NE (c) in ShA (hashed columns) and ShA (filled columns), sensitive (solid-gray columns, respectively $n=19$ and 15) and resistant (solid-black columns, $n=3$ and 9) rats. The data are mean levels $( \pm$ SEM.) of both left and right hemisphere. 5-HT and DA utilization indices were determined by the ratio of the respective metabolite of the parent transmitter. Significant differences between sensitive and resistant *Fischer's LSD $P<0.05$.

Rats with a limited cocaine SA history having received icv 5,7-DHT infusions had significant depletions of 5-HT compared to sham animals of between 87 and $92 \%$ in prefrontal cortical and striatal areas, as well as in the amygdala (effect of group: $\mathrm{F}(1,46)=15, P<0.0001$ ). There were no significant reductions in DA or NA content (Supplementary Materials Table 1).

Although completing similar numbers of seeking cycles under baseline conditions, rats with forebrain 5-HT depletion completed significantly more seeking cycles than sham operated controls when the intermittent punishment contingency was introduced (interaction punishment $\times$ group: $\mathrm{F}(1,46)=11.1, P=0.002$ ) (Figure $4 \mathrm{~b}$ ). There were no differences between the lesioned and sham groups in the motivational measures for cocaine (break points under the progressive ratio schedule of reinforcement and number of responses in the seeking probe test Supplementary Material Table 2) or in the level of nose-poke responding for sucrose. There were significant correlations between the normalized levels of serotonin in the amygdala, the striatum and the prefrontal cortex and the seeking rate during the last four days of punishment $(\rho \mathrm{s}(46)>-0.28$;
$P<0.05)$ whereas the nose-poke rate for the same period was not correlated with the level of serotonin in any of the structures.

Rats with forebrain 5-HT depletion did not differ significantly from sham control rats in their locomotor reactivity to mild footshock (number of line crosses during shock in the conditioning environment), but did show lower levels of conditioned freezing in response to the CS previously associated with mild footshock (effect of resistance: $\mathrm{F}(19,418)=2 ; P=0.008$ ) (Supplementary Materials Figure 6).

Injection i.p. of the 5-HT2C receptor agonist $\mathrm{mCPP}$ restored sensitivity to punishment (effect of dose: $\mathrm{F}(3,66)=12.4, P<0.001)$ but only in 5,7-DHT-lesioned rats (interaction group $\mathrm{x}$ dose: $\mathrm{F}(3,66)=3.3 ; P=0.03$ ), resulting in a dose-dependent decrease in the number of seeking cycles completed $(P<0.001)$. The effect was significant at doses of 0.3 and $1 \mathrm{mg} / \mathrm{kg}(P \mathrm{~s}<0.001)$, thereby abolishing the initial differences between groups $(P s<0.025)$ (Figure $4 c)$. The highest dose of $\mathrm{mCPP}$ also resulted in a decrease in responding for sucrose (effect of dose: $\mathrm{F}(3,66)=5.3$, $P=0.02$ ), but only in the sham-operated controls (interaction: $\mathrm{F}(3,66)=2.6, P=0.05)$. 
Figure $4 \mathrm{~d}$ shows that the 5-HT2C receptor antagonist, SB242084 and the 5-HT2A receptor antagonist, M100907 had markedly different effects on cocaine seeking under the intermittent punishment contingency in non-lesioned rats with a limited cocaine SA history (interaction dose $\times$ drug: $\mathrm{F}(1,51)=17.6 ; P=0.001)$. The $5-\mathrm{HT} 2 \mathrm{C}$ receptor antagonist SB242084 resulted in a significant dose-dependent increase in the number of seeking cycles completed under punishment compared to vehicle-treated rats (Fischer's LSD
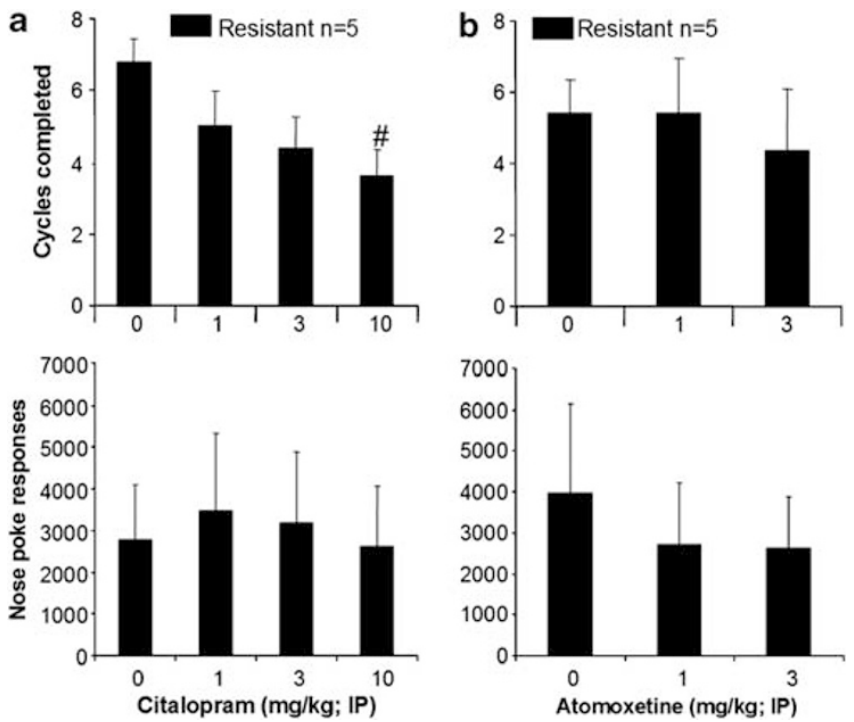

Figure 3 Reinstatement of punishment sensitivity by selective serotonin reuptake inhibition. (a) Effects of increasing doses of citalopram or (b) atomoxetine on the number of seeking cycles completed under punishment (upper panels) or the concomitant nose-poke responding for sucrose (lower panels) in resistant animals, mean \pm SEM of five animals. Significantly different from saline: "Fischer's LSD $P<0.05$.
Ps $<0.02)$. Treatment with the $5-\mathrm{HT} 2 \mathrm{~A}$ receptor antagonist M100907, in contrast, had no effect or actually resulted in a small decrease in the number of seeking cycles under punishment $(P=0.04)$. Neither drug had any effect on concomitant responding for sucrose $(\mathrm{Fs}<1.36)$.

\section{DISCUSSION}

The results of these experiments show that the great majority of a population of rats with escalated cocaine intake is able to withhold cocaine-seeking responses effectively achieving abstinence - when there is an intermittent risk of punishment. However, under these circumstances, $20 \%$ of rats continued to seek cocaine compulsively, whereas only $6 \%$ of rats with controlled cocaine intake (that had not escalated) and no rats with a very limited drug history having recently acquired cocaine seeking did so. The present results thereby extend our earlier observations showing a nonlinear increase in the proportion of animals resistant to punishment that seek cocaine compulsively as a function of their cocaine-taking history (Deroche-Gamonet et al, 2004; Pelloux et al, 2007). These results also provided us with the opportunity in this vulnerable subgroup of rats to investigate the neural and psychological mechanisms underlying the development of compulsive drug seeking, described clinically in addicted individuals as occurring in the face of conflict with other incentives, including the fear of punishment (Waldorf et al, 1991; Ersche et al, 2011).

Neurochemical analyses revealed that compulsive rats had significantly lower 5-HT:5-HIAA ratios in dorsal and ventral prefrontal cortical areas as well as in the amygdala, and in the ventral and dorsal striatum. Changes in DA utilization were more neuroanatomically restricted, being significantly decreased only in the dorsal striatum. These

a
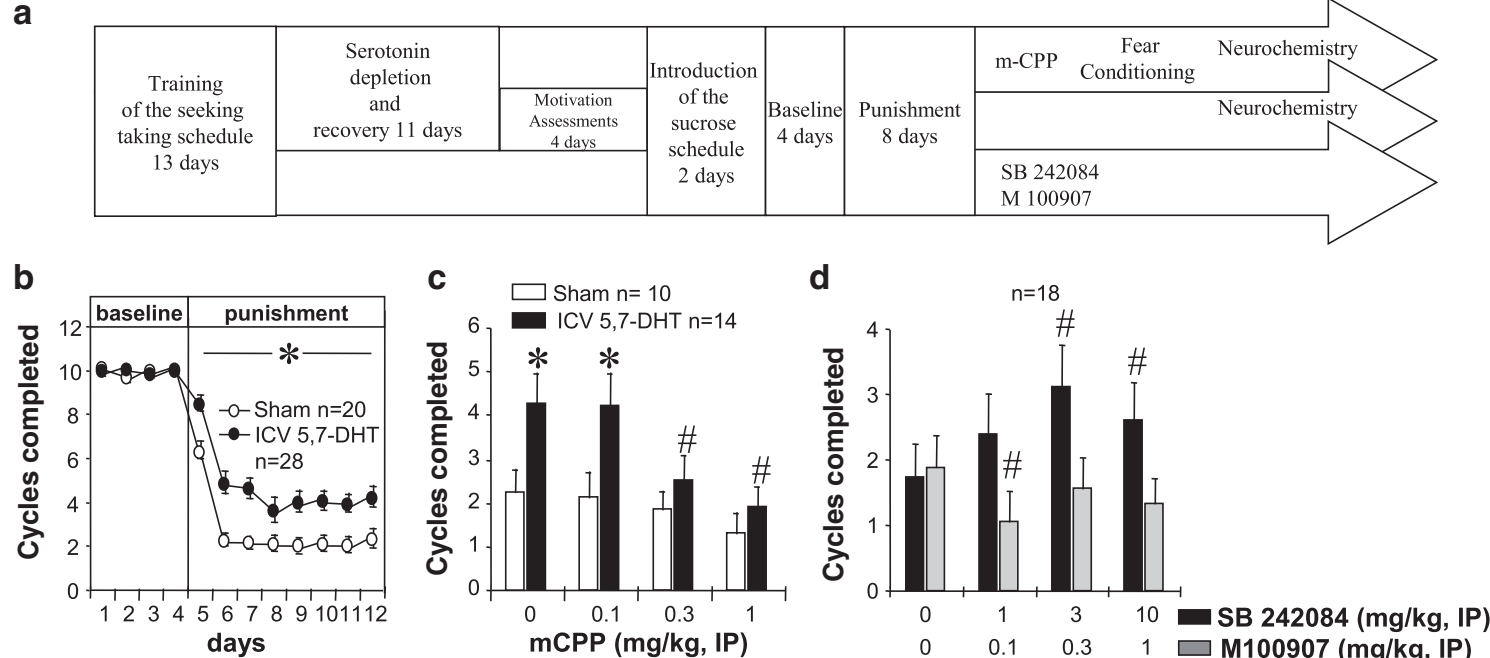

Figure 4 Bidirectional modulation of compulsive cocaine seeking by serotoninergic manipulations (a) Timeline of the experiment; (b) number of seeking cycles completed before (baseline) and during punishment of cocaine-seeking responding; (c) the effects of increasing doses of mCPP on the number of seeking cycles completed under punishment in the ICV 5,7-DHT and sham-operated control animals: Mean \pm SEM of 20 and 28 animals. *: significant differences between sham and ICV 5,7-DHT, Fischer LSD $P<0.03$ \#: significant differences from vehicle condition, Fischer's LSD $P<0.00$ I (d) The effect of increasing doses of SB242084 and MI00907 on the number of seeking cycles completed under punishment in animals after a short history of cocaine taking. Mean \pm SEM of 18 animals: significant differences from vehicle condition \#: Fischer LSD $P<0.04$. 
changes in 5-HT and DA utilization, which clearly differentiated compulsive and punishment-sensitive groups despite their common drug history, could not therefore be attributed to different levels of cocaine exposure, nor did they correlate with the total amount of drug consumed during the free access sessions.

Reductions in indices of DA transmission have been reported previously in drug-addicted populations, including reduced DA levels (Wilson et al, 1996) and dopamine D2 receptors in the striatum (Volkow et al, 2001). Similarly, a reduction in striatal dopamine D2 receptors and an increase in striatal DA transporters have also been shown to follow chronic, but not acute, cocaine SA in monkeys (Porrino et al, 2007; Letchworth et al, 2001), suggesting that they may be a consequence of an extended cocaine-taking history. Decreased 5-HT levels in prefrontal cortex have also been reported in stimulant-addicted individuals studied post mortem (Wilson et al, 1996), with subsequent research providing evidence that this may be causally related to their cognitive and behavioral deficits. Thus, impairments in probabilistic decision-making (Rogers et al, 1999a), but most especially increased perseverative responding by cocaine-addicted individuals in a reversal learning task (Ersche et al, 2008) were reproduced in healthy volunteers by tryptophan depletion (Rogers et al, 1999a; Rogers et al, 1999b). Perseverative responding in reversal learning, a form of compulsive behavior, has also been shown to follow disruption of prefrontal cortical-dorsal striatal circuitry in rats (Castañé et al, 2010) and monkeys (Clarke et al, 2008), and to be correlated with activity in this circuitry in human subjects (Cools et al, 2002). It may therefore be hypothesized that disruption of serotoninergic transmission within fronto-striatal circuitry may be especially important for the development of compulsive cocaine seeking.

The compulsive cocaine seeking that emerged in rats after an extended cocaine history was remediated by acute treatment with the SSRI, citalopram, but not with the selective NARI, atomoxetine, consistent with the predictions based on the neurochemical data. This result indicates both the possible causal involvement of reduced 5-HT transmission in the compulsive cocaine-seeking phenotype and the therapeutic potential of reversing this deficit and thereby the propensity to seek cocaine, perhaps especially in the now-recognized subgroup of cocaine abusers who show greater compulsivity in their cocaine use (Ersche et al, 2011) This indication of clinical utility in the acute effect of citalopram to reduce compulsive cocaine seeking is, however, tempered by the outcome of several clinical trials involving more chronic SSRI treatment, which have failed to show efficacy in the treatment of cocaine addiction (Covi et al, 1995; Grabowski et al, 1995; Batki et al, 1996). This may reflect the well-known impact of adaptations in 5-HT receptors and transmission by chronic SSRI treatments (eg Sanders-Bush et al, 1989) that counteract the beneficial effect of 5-HT reuptake inhibition. However, an alternative explanation (Vayalapalli et al, 2011) is that most clinical studies have employed the low doses of SSRI used to treat depression in humans (and with some success when there is a co-occurring mood disorder), and not the doses that have been used successfully to treat obsessive compulsive disorder, which are considerably higher (eg Moeller et al, 2007), and likely more comparable to the doses used in the present study. A high-dose citalopram trial for cocaine dependence is currently being undertaken to test this prediction (see Vayalapalli et al, 2011).

The hypothesis of a causal involvement of 5-HT in compulsive cocaine seeking was further supported by the observation that rats with forebrain selective 5-HT depletion withheld significantly fewer of their drug-seeking responses when the intermittent punishment contingency was introduced after a short history of cocaine SA, at a time when no intact rats displayed compulsive drug seeking. This increased tendency to seek cocaine compulsively following 5-HT depletion was completely reversed by treatment with the 5-HT2C receptor agonist, mCPP. Conversely, intact (ie, non-5-HT lesioned) rats treated with the 5-HT2C receptor antagonist under the same, short cocaine-taking history conditions increased their cocaine seeking under punishment to an extent similar to that seen following forebrain 5-HT depletion, whereas the 5-HT2A receptor antagonist was without effect or tended to reduce cocaine seeking.

Taken together, these data indicate that reductions in 5HT transmission seen after a long cocaine SA history are causally related to the emergence of compulsive cocaine seeking in a vulnerable sub-group of rats, and that altered 5-HT transmission through 5-HT2C receptors may be particularly implicated in this process.

Although forebrain 5-HT depletion resulted in persistent cocaine seeking under punishment, the behavioral phenotype of these rats also differed somewhat from the compulsive animals that emerged after an extended cocaine-taking history. The acutely 5-HT depleted rats showed some reduction in conditioned fear (measured as time spent freezing to a fear CS), whereas the compulsive cocaine-seeking group did not. However it is unlikely that reductions in fear led to compulsive cocaine seeking. Only the rate of the punished cocaine-seeking response, and not the rate of unpunished, concomitant nose-poke responding for sucrose, was correlated with the forebrain levels of serotonin. In addition, in the compulsive subgroup that emerged after an extended cocaine SA history, there were no differences in conditioned fear, nor in unconditioned responses to the aversive, mild foot shock stimulus. It is perhaps not surprising that an acutely induced, selective 5HT lesion in rats with a limited cocaine SA history did not recapitulate the full range of changes seen after extended and escalated exposure to the drug (which was also associated with decreased striatal DA turnover) in which resistance to punishment develops over time. It is likely that a full understanding of the relationship between the 5-HT pathology and compulsive cocaine seeking requires investigation, probably involving timed interventions in discrete neural loci that are innervated by median or dorsal raphe projection systems. For example, serotonergic pathways arising from the dorsal raphe nucleus have specifically been implicated in the control of behavior under punishment (Thiébot et al, 1983), whereas those emanating from the median raphe have been more implicated in conditioned fear (Avanzi et al, 2003; Melik et al, 2000); both systems would have been compromised by i.c.v. 5,7-DHT.

As the proportion of compulsive animals increased with the extent of exposure to cocaine, it may be hypothesized that emerging resistance to the intermittent punishment of cocaine seeking is a consequence of chronic cocaine-induced 
reductions in 5-HT (and DA) transmission. Consistent with such a view is the finding that cocaine sensitization treatment (Schoenbaum et al, 2004) or cocaine SA (Calu et al, 2007), both resulted in perseverative (compulsive) responding in reversal learning tasks. However, it has also been shown that resistance to the punishment of cocaine seeking and the propensity to relapse after abstinence are predicted by pre-existing (ie, pre-cocaine exposure) individual differences in impulsivity (Belin et al, 2008; Economidou et al, 2009). Impulsivity can be induced by forebrain 5-HT depletion (Harrison et al, 1997); individual differences in impulsivity are correlated with the level of extracellular 5-HT within the prefrontal cortex (Dalley et al, 2002), and treatment with a 5-HT2C receptor antagonist can increase impulsivity (Winstanley et al, 2004). Thus, a preexisting deficit in behavioral inhibition (ie, impulsivity), which is itself associated with loss of control over cocaine intake (Dalley et al, 2007), may exacerbate cocaine-induced deficits in 5-HT transmission in the prefrontal corticalstriatal systems that together lead to compulsive cocaine seeking

None of the serotonin manipulations affected unpunished responding for sucrose, revealing the preferential involvement of serotonin in responding under conditions of appetitive and aversive response conflict. However, an increase in the motivation for cocaine following prolonged use (Robinson and Berridge, 1993), in part as a result of reduced serotonin transmission, may also have contributed to compulsive cocaine seeking. Thus, both depletion of central 5-HT (Loh and Roberts, 1990) or treatment with a 5-HT2C receptor antagonist (Higgins and Fletcher, 2003) have been shown to increase the SA of cocaine and to increase the break point under a progressive ratio schedule. Furthermore, it has been suggested that 5-HT2C receptor agonists may be useful in the treatment of substance abuse disorders by decreasing the rewarding effects of cocaine (Higgins \& Fletcher, 2003). Variation in the level of resistance to punishment among all animals was indeed correlated with baseline seeking performance and two motivational measures, break points under a progressive ratio schedule of reinforcement and seeking responses in probe tests, both conducted at the time of introduction of the punishment contingency. However, compulsive cocaine seeking can also develop in the absence of increased motivational measures, or escalated cocaine intake, or both (Vanderschuren \& Everitt, 2004; Deroche-Gamonet et al, 2004; Pelloux et al, 2007; Belin et al, 2008). Activation of the stress system by foot shock can reinstate responding for cocaine in LgA rats, an effect suggested to be mediated by modulation of ventral tegmental area DA neuron activity (Blacktop et al, 2011), and thus indicating an additional mechanism underlying the propensity to seek cocaine, although it is unclear whether this mechanism is engaged under conditions of conflict.

Thus, heterogeneous processes are likely engaged after extended or escalated cocaine intake to determine the propensity to seek the drug when the increased motivation to do so is also in conflict with unpredictable aversive outcomes. The motivation to obtain access to cocaine is not necessarily constrained by the motivation to avoid shock in a conflict situation, but both may be modulated by alterations in 5-HT transmission. Indeed, in human decision-making, it has recently been demonstrated that acute depletion of brain 5-HT altered the mechanism by which rewards and punishments were compared, by influencing the encoding of both reward value and punishment and leading to what was termed choice persistence through modulation of activity in fronto-striatal systems (Seymour et al, 2012). Thus, a pro-5-HT treatment with an SSRI such as citalopram, that both increases the impact of punishment and decreases the value of cocaine, may be especially effective in targeting both processes.

In summary, the results of the present experiments demonstrate that compulsive cocaine seeking emerges in a vulnerable subpopulation of animals after an escalated cocaine-taking history. These compulsive animals had a marked reduction in forebrain 5-HT that was shown causally to be related to their inability to abstain from responding for cocaine in the face of intermittent and unpredictable punishment. Moreover, acute treatment with an SSRI ameliorated the propensity for compulsive cocaine seeking, suggesting the clinical treatment potential of targeting the serotonin deficit to reduce the compulsion to seek cocaine.

\section{ACKNOWLEDGEMENTS}

This work was supported by the United Kingdom Medical Research Council (MRC) Grant to BJE (G9536855) and was conducted within the MRC/Wellcome Trust Behavioural and Clinical Neuroscience Institute. We would like to thank Prof. Trevor Robbins, Prof. Anthony Dickinson, Dr Sietse Jonkman and Dr Jennifer Murray for their insightful comments and Dr Adam Mar for help with the statistical analysis of the data and valuable discussion.

\section{DISCLOSURE}

The authors declare no conflict of interest.

\section{REFERENCES}

Ahmed SH, Koob GF (1998). Transition from moderate to excessive drug intake: change in hedonic set point. Science 282: 298-300.

American Psychiatric Association (2000). Diagnostic and Statistical Manual of Mental Disorders 4th edn. American Psychiatric Association: Washington, DC.

Anthony JC, Warner LA, Kessler RC (1994). Comparative epidemiology of dependence on tobacco, alcohol, controlled substances, and inhalants. Exp Clin Psychopharmacol 2: 244-268.

Avanzi V, Silva RC, Macedo CE, Brandão ML (2003). 5-HT mechanisms of median raphe nucleus in the conditioned freezing caused by light/foot-shock association. Physiol Behav 78: $471-477$.

Batki SL, Washburn AM, Delucchi K, Jones RT (1996). A controlled trial of fluoxetine in crack cocaine dependence. Drug Alcohol Depend 41: 137-142.

Belin D, Mar AC, Dalley JW, Robbins TW, Everitt BJ (2008). High impulsivity predicts the switch to compulsive cocaine-taking. Science 320: 1352-1355.

Björklund A, Baumgarten HG, Lachenmayer L, Rosengren E (1975). Recovery of brain noradrenaline after 5,7-dihydroxytryptamine-induced axonal lesions in the rat. Cell Tissue Res 161: 145-155. 
Blacktop JM, Seubert C, Baker DA, Ferda N, Lee G, Graf EN et al (2011). Augmented cocaine seeking in response to stress or CRF delivered into the ventral tegmental area following long-access self-administration is mediated by CRF receptor type 1 but not CRF receptor type 2. J Neurosci 31: 11396-11403.

Calu DJ, Stalnaker TA, Franz TM, Singh T, Shaham Y, Schoenbaum $G$ (2007). Withdrawal from cocaine self-administration produces long-lasting deficits in orbitofrontal-dependent reversal learning in rats. Learn Mem 14: 325-328.

Castañé A, Theobald DE, Robbins TW (2010). Selective lesions of the dorsomedial striatum impair serial spatial reversal learning in rats. Behav Brain Res 210: 74-83.

Clarke HF, Robbins TW, Roberts AC (2008). Lesions of the medial striatum in monkeys produce perseverative impairments during reversal learning similar to those produced by lesions of the orbitofrontal cortex. J Neurosci 28: 10972-10982.

Cools R, Clark L, Owen AM, Robbins TW (2002). Defining the neural mechanisms of probabilistic reversal learning using event-related functional magnetic resonance imaging. J Neurosci 22: 4563-4567.

Cooper A, Barnea-Ygael N, Levy D, Shaham Y, Zangen A (2007). A conflict rat model of cue-induced relapse to cocaine seeking. Psychopharmacology 194: 117-125.

Covi L, Hess JM, Kreiter NA, Haertzen CA (1995). Effects of combined fluoxetine and counseling in the outpatient treatment of cocaine abusers. Am J Drug Alcohol Abuse 21: 327-344.

Dalley JW, Theobald DE, Eagle DM, Passetti F, Robbins TW (2002). Deficits in impulse control associated with tonically-elevated serotonergic function in rat prefrontal cortex. Neuropsychopharmacology 26: 716-728.

Dalley JW, Fryer TD, Brichard L, Robinson ES, Theobald DE, Lääne K et al (2007). Nucleus accumbens D2/3 receptors predict trait impulsivity and cocaine reinforcement. Science 315: $1267-1270$.

Deroche-Gamonet V, Belin D, Piazza PV (2004). Evidence for addiction-like behavior in the rat. Science 305: 1014-1017.

Economidou D, Pelloux Y, Robbins TW, Dalley JW, Everitt BJ (2009). High impulsivity predicts relapse to cocaine-seeking after punishment-induced abstinence. Biol Psychiatry 65: 851-856.

Ersche KD, Barnes A, Jones PS, Morein-Zamir S, Robbins TW, Bullmore ET (2011). Abnormal structure of frontostriatal brain systems is associated with aspects of impulsivity and compulsivity in cocaine dependence. Brain 134: 2013-2024.

Ersche KD, Roiser JP, Robbins TW, Sahakian BJ (2008). Chronic cocaine but not chronic amphetamine use is associated with perseverative responding in humans. Psychopharmacology 197: 421-431.

Grabowski J, Rhoades H, Elk R, Schmitz J, Davis C, Creson D et al (1995). Fluoxetine is ineffective for treatment of cocaine dependence or concurrent opiate and cocaine dependence: two placebo-controlled double-blind trials. J Clin Psychopharmacol 15: $163-174$.

Harrison AA, Everitt BJ, Robbins TW (1997). Central 5-HT depletion enhances impulsive responding without affecting the accuracy of attentional performance: interactions with dopaminergic mechanisms. Psychopharmacology 133: 329-342.

Heyne A, Wolffgramm J (1998). The development of addiction to d-amphetamine in an animal model: same principles as for alcohol and opiate. Psychopharmacology 140: 510-518.

Higgins GA, Fletcher PJ (2003). Serotonin and drug reward: focus on 5-HT2C receptors. Eur J Pharmacol 480: 151-162.

Hodos W (1961). Progressive ratio as a measure of reward strength. Science 134: 943-944.

Jacobsen LK, Staley JK, Malison RT, Zoghbi SS, Seibyl JP, Kosten TR et al (2000). Elevated central serotonin transporter binding availability in acutely abstinent cocaine-dependent patients. $\mathrm{Am}$ J Psychiatry 157: 1134-1140.

Letchworth SR, Nader MA, Smith HR, Friedman DP, Porrino LJ (2001). Progression of changes in dopamine transporter binding site density as a result of cocaine self-administration in rhesus monkeys. J Neurosci 21: 2799-2807.

Little KY, McLaughlin DP, Zhang L, Livermore CS, Dalack GW, McFinton PR et al (1998). Cocaine, ethanol, and genotype effects on human midbrain serotonin transporter binding sites and mRNA levels. Am J Psychiatry 155: 207-213.

Loh EA, Roberts DC (1990). Break-points on a progressive ratio schedule reinforced by intravenous cocaine increase following depletion of forebrain serotonin. Psychopharmacology 101: 262-266.

Melik E, Babar-Melik E, Ozgünen T, Binokay S (2000). Median raphe nucleus mediates forming long-term but not shortterm contextual fear conditioning in rats. Behav Brain Res 112: 145-150.

Moeller FG, Schmitz JM, Steinberg JL (2007). Citalopram combined with behavioral therapy reduces cocaine use: A double-blind placebo-controlled trial. Am J Drug Alcohol Abuse 33: 367-378.

Panlilio LV, Thorndike EB, Schindler CW (2003). Reinstatement of punishment-suppressed opioid self-administration in rats: an alternative model of relapse to drug abuse. Psychopharmacology 168: 229-235.

Paxinos G, Watson C (1996). The Rat Brain in Stereotaxic Coordinate Academic Press: New York.

Pelloux Y, Everitt BJ, Dickinson A (2007). Compulsive drug seeking by rats under punishment: effects of drug taking history. Psychopharmacology 194: 127-137.

Porrino LJ, Smith HR, Nader MA, Beveridge TJ (2007). The effects of cocaine: a shifting target over the course of addiction. Prog Neuropsychopharmacol Biol Psychiatry 31: 1593-1600.

Porter RH, Benwell KR, Lamb H, Malcolm CS, Allen NH, Revell DF et al (1999). Functional characterization of agonists at recombinant human 5-HT2A, 5-HT2B and 5-HT2C receptors in CHO-K1 cells. Br J Pharmacol 128: 13-20.

Robinson TE, Berridge KC (1993). The neural basis of drug craving: an incentive-sensitization theory of addiction. Brain Res Brain Res Rev 18: 247-291.

Rogers RD, Everitt BJ, Baldacchino A, Blackshaw AJ, Swainson R, Wynne $\mathrm{K}$ et al (1999a). Dissociable deficits in the decisionmaking cognition of chronic amphetamine abusers, opiate abusers, patients with focal damage to prefrontal cortex, and tryptophan-depleted normal volunteers: evidence for monoaminergic mechanisms. Neuropsychopharmacology 20: 322-339.

Rogers RD, Blackshaw AJ, Middleton HC, Matthews K, Hawtin K, Crowley C et al (1999b). Tryptophan depletion impairs stimulusreward learning while methylphenidate disrupts attentional control in healthy young adults: implications for the monoaminergic basis of impulsive behaviour. Psychopharmacology 146: 482-491.

Sanders-Bush E, Breeding M, Knoth K, Tsutsumi M (1989). Sertraline-induced desensitization of the serotonin 5HT-2 receptor transmembrane signaling system. Psychopharmacology 99: 64-69.

Schoenbaum G, Saddoris MP, Ramus SJ, Shaham Y, Setlow B (2004). Cocaine-experienced rats exhibit learning deficits in a task sensitive to orbitofrontal cortex lesions. Eur J Neurosci 19: 1997-2002.

Seymour B, Daw ND, Roiser JP, Dayan P, Dolan R (2012). Serotonin selectively modulates reward value in human decision-making. J Neurosci 32: 5833-5842.

Shaham Y, Shalev U, Lu L, De Wit H, Stewart J (2003). The reinstatement model of drug relapse: history, methodology and major findings. Psychopharmacology 168: 3-20. 
Spanagel R, Holter SM (1999). Long-term alcohol self-administration with repeated alcohol deprivation phases: an animal model of alcoholism? Alcohol Alcohol 34: 231-243.

Thiébot MH, Hamon M, Soubrié P (1983). The involvement of nigral serotonin innervation in the control of punishmentinduced behavioral inhibition in rats. Pharmacol Biochem Behav 19: 225-229.

Vanderschuren LJ, Everitt BJ (2004). Drug seeking becomes compulsive after prolonged cocaine self-administration. Science 305: 1017-1019.

Vayalapalli S, Vaughn M, Salles-Shahid K, Byrd-Sellers J, Drexler K (2011). High-dose citalopram for cocaine dependence in veteran population-a pilot project. Am J Addict 20: 485-486.

Volkow ND, Chang L, Wang GJ, Fowler JS, Ding YS, Sedler M et al (2001). Low level of brain dopamine D-2 receptors in methamphetamine abusers: association with metabolism in the orbitofrontal cortex. Am J Psychiatry 158: 2015-2021.
Waldorf D, Reinarman C, Murphy S (1991). Cocaine changes: the experience of using and quitting. Temple University Press: Philadelphia.

Wilson JM, Kalasinsky KS, Levey A, Bergeron C, Reiber G, Anthony $\mathrm{RM}$ et al (1996). Striatal dopamine nerve terminal markers in human, chronic methamphetamine users. Nat Med 2: 699-703.

Winstanley CA, Theobald DE, Dalley JW, Glennon JC, Robbins TW (2004). 5-HT2A and 5-HT2C receptor antagonists have opposing effects on a measure of impulsivity: interactions with global 5HT depletion. Psychopharmacology 176: 376-385.

This work is licensed under the Creative

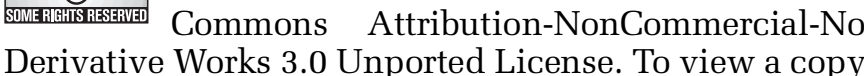
of this license, visit http://creativecommons.org/licenses/by-nc-nd/3.0/

Supplementary Information accompanies the paper on the Neuropsychopharmacology website (http://www.nature.com/npp) 